\title{
Direct 3D Bioprinting of Perfusable Vascular Constructs Using a Blend Bioink
}

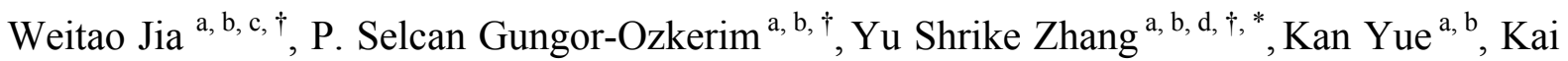
Zhu ${ }^{\text {a, b, e }}$, Wanjun Liu ${ }^{\text {a, b }}$, Qingment Pi ${ }^{\text {a, b }}$, Batzaya Byambaa ${ }^{\text {a, b }}$, Mehmet Remzi Dokmeci ${ }^{\text {a, b, }}$ d, Su Ryon Shin ${ }^{\text {a, b, d, *, Ali Khademhosseini }}{ }^{\text {a, b, d, f, g, * }}$

${ }^{a}$ Biomaterials Innovation Research Center, Division of Biomedical Engineering, Department of Medicine, Brigham and Women's Hospital, Harvard Medical School, Cambridge, MA 02139, USA.

${ }^{b}$ Harvard-MIT Division of Health Sciences and Technology, Massachusetts Institute of Technology, Cambridge, MA 02139, USA.

${ }^{c}$ Department of Orthopedic Surgery, Shanghai Jiaotong University Affiliated Sixth People's Hospital, Shanghai Jiaotong University, Shanghai 200233, P.R. China.

${ }^{\mathrm{d}}$ Wyss Institute for Biologically Inspired Engineering, Harvard University, Boston, MA 02115, USA.

${ }^{\mathrm{e}}$ Department of Cardiac Surgery, Zhongshan Hospital, Fudan University, Shanghai Institute of Cardiovascular Disease, Shanghai 200032, PR China.

${ }^{\mathrm{f}}$ Department of Bioindustrial Technologies, College of Animal Bioscience and Technology, Konkuk University, Seoul 143-701, Republic of Korea.

g Department of Physics, King Abdulaziz University, Jeddah 21569, Saudi Arabia.

${ }^{\dagger}$ These authors contributed equally as first author to this work.

* Corresponding authors: alik@bwh.harvard.edu (A.K.); sshin4@partners.org (S.R.S.); yszhang@research.bwh.harvard.edu (Y.S.Z.)

Running headline: 3D Bioprinting of Perfusable Vascular Constructs 


\begin{abstract}
Despite significant technological advancement in tissue engineering, challenges still exist towards the development of complex and fully functional tissue constructs that mimic their natural counterparts. To address these challenges, bioprinting has emerged as an enabling technology to create highly organized three-dimensional (3D) vascular networks within engineered tissue constructs to promote the transport of oxygen, nutrients, and waste products, which can hardly be realized using conventional microfabrication techniques. Here, we report the development of a versatile 3D bioprinting strategy that employs biomimetic biomaterials and an advanced extrusion system to deposit perfusable vascular structures with highly ordered arrangements in a single-step process. In particular, a specially designed cellresponsive bioink consisted of gelatin methacryloyl (GelMA), sodium alginate, and 4-arm poly(ethylene glycol)-tetra-acrylate (PEGTA) were used in combination with a multilayered coaxial extrusion systems to achieve direct 3D bioprinting. This blend bioink could be first ionically crosslinked by calcium ions followed by covalent photocrosslinking for GelMA and 4-arm PEGTA to form stable constructs. The rheological properties of the bioink and mechanical strengths of the resulting constructs were tuned by the introduction of a 4-arm PEGTA, which facilitated the precise deposition of complex multilayered 3D perfusable hollow tubes. This blend bioink also displayed favorable biological characteristics that supported the spreading and proliferation of encapsulated endothelial and stem cells in the bioprinted constructs, leading to the formation of biologically relevant, highly organized, perfusable vessels. These characteristics make this novel 3D bioprinting technique superior to conventional microfabrication or sacrificial templating approaches for fabrication of the perfusable vasculature. We envision that our advanced bioprinting technology may also have significant potentials in engineering large-scale vascularized tissue constructs towards applications in organ transplantation and repair.
\end{abstract}

Keywords: 3D bioprinting, perfusable hollow tube, bioink, endothelial cells, mesenchymal stem cells 


\section{Introduction}

Tissue engineering holds great promise as an alternative therapy by creating functional tissue constructs that can reestablish the structure and function of the damaged tissues [1-4]. Despite significant advancement in the field of regenerative medicine and tissue engineering achieved during the past few decades, fabrication of complex and functional tissue constructs that mimic their natural counterparts still remains a challenge [5-7]. In order to promote the functions of the engineered tissues, the development of interconnected 3D vascular networks within the tissue constructs plays a critical role [8-10]. To date, numerous microfabrication approaches have been devised to form randomly assembled microvessels within engineered tissue constructs via micromolding, biotextiles, and photolithography [11-17]. Despite great progress in these approaches, many are still focused on angiogenesis induction, which typically requires substantial amounts of time to establish. Furthermore, these engineered tissues are limited by their dependence on diffusion of the host's nutrient and oxygen due to the initial lacking of integration within the host body post implantation. Therefore, the creation of perfusable vasculature within an engineered tissue construct is expected to solve these problems as it immediately allows for blood transport. Such perfusion can provide sufficient supplies of oxygen and nutrients and meanwhile remove waste products, avoiding the potential formation of necrotic regions within the bulk tissues [18, 19].

To this end, bioprinting has recently emerged as a promising technology for fabrication of highly organized 3D vascular networks through controlled deposition of cells and biomaterials [15, 20-25]. Most of these techniques are based on sacrificial bioprinting, where a templating bioink is firstly deposited and embedded in a hydrogel matrix, followed by removal of the bioink to obtain the hollow vessel-like structures [22-25]. The channels can then be populated with endothelial cells to further introduce the biological functions. Alternatively, agarose rods was printed concomitantly as a temporary building blocks of a molding template to support the fusion of multicellular spheroids or cylinders into vessel like structure $[20,26]$. Due to the complexity involved in the procedure for the sacrificial bioprinting, the size, morphology, and functionality of the obtained vessel-like structures may be limited. Despite recent technological advancements in the development of multilayered coaxial extrusion systems combined with a wet spinning platform has rendered it possible to 
directly fabricate hollow hydrogel tubes, generation of complex 3D cell-laden hollow structures is still not easily achievable and have not been demonstrated [27-30]. Therefore, methods that are capable of directly bioprinting perfusable vasculature with highly organized spatial distribution in a single step are strongly desired.

Cell responsive bioink constitutes another critical component in bioprinting perfusable hollow tubes. A variety of hydrogel materials, such as gelatin, collagen, hyaluronic acid, alginate, and poly(ethylene glycol) (PEG), have been used individually as bioink due to their biocompatibility and sufficient viscosity for bioprinting with high fidelity [31-33]. However, none of these materials alone offers properties suitable for direct bioprinting of perfusable hollow tubes. For example, the extracellular matrix (ECM)-based biomaterials, including gelatin, collagen, and hyaluronic acid, suffer from limited mechanical strength [34-37], which if used alone, would not be able to support the weight of the hollow tubes and inevitably lead to collapse of the structures. On the other hand, alginate or PEG along, although mechanically tough, does not possess cell-adhering moieties required for spreading, motility, and proliferation of the encapsulated cells [38]. This is exemplified in a few recent works where hollow tubes resembling blood vessels were direct extruded or bioplotted but limited cellular responses could be achieved [27-30]. Therefore, blending of several biomaterials capable of forming hydrogels have been increasingly developed to use as bioinks, the biological and mechanic properties of which can be custom-tailored according to different requirements [39].

Herein, we present a convenient strategy capable of generating highly organized perfusable vascular structures using a simple, one-step 3D bioprinting technique based on a multilayered coaxial nozzle device in combination with our specially designed cell-laden blend bioink. In particular, we engineered a blend bioink based on gelatin methacryloyl (GelMA) [40], sodium alginate, and 4-arm poly(ethylene glycol)-tetra-acrylate (PEGTA), which not only possesses desired rheological properties and printability as the bioink, but also shows sufficient mechanical strength and bioactivity of the resulting constructs. The alginate component enabled fast ionic crosslinking and shape maintenance of the initially bioprinted perfusable tubes through co-delivery of $\mathrm{CaCl}_{2}$ solution [41-43], while the morphologies of the constructs could be permanently fixed by subsequent photocrosslinking of the GelMA 
and 4-arm PEGTA components. Such a dual-stage crosslinking mechanism is comparable with that reported in our previous work [44], but both the printhead setup and the composition of the bioink have been significantly modified to achieve optimal bioprinting of hollow vascular microfibers. For example, a tri-layered coaxial nozzle is the key to successful deposition of perfusable structures, and the 4-arm PEGTA component used in this study is also superior to commonly used linear PEG derivatives through enhanced crosslinking density and thus increased mechanical strength while maintaining the beneficial porous structure, due to its branched tetravalent chemical structure and multiple active crosslinking sites. This porous structure could induce better cell growth and spreading compared to hydrogels based on conventional PEG-diacrylate, which was proved in our previous study [45]. By combining benefits of both natural and synthetic biomaterials, this unique bioink formulation displayed favorable biochemical characteristics for survival and proliferation of encapsulated vascular cells and tunable mechanical properties for bioprinting complex perfusable vascular constructs, inducing the formation of biologically relevant, highly organized, intact vessels. These characteristics have made this approach superior to current methods for fabrication of the vasculature, indicating its potential applications in engineering large-scale vascularized tissue constructs for organ transplantation and repair.

\section{Materials and methods}

\subsection{Materials}

Sodium alginate $(\mathrm{Mw}=33 \mathrm{kDa}$; low viscosity), gelatin from porcine skin (type-A, 300 bloom), methacrylic anhydride (MW 154.16), 2-hydroxy-1-[4-(2-hydroxyethoxy) phenyl]-2methyl-1-propanone (Irgacure 2959), calcium chloride $\left(\mathrm{CaCl}_{2}\right)$, bovine serum albumin (BSA), and ethylenediaminetetraacetic acid (EDTA) were purchased from Sigma-Aldrich (St. Louis, MO, USA). 4-arm PEGTA (Mw=20 kDa) was purchased from JenKem Technology (Beijing, China). Transforming growth factor- $\beta 1$ (TGF- $\beta 1$ ), Fetal bovine serum (FBS), alpha minimum essential medium ( $\alpha$-MEM), phosphate buffered saline (PBS), 2-[4-(2hydroxyethyl) piperazin-1-yl] ethane sulfonic acid (HEPES buffer, $25 \mathrm{mM}, \mathrm{pH}$ 7.4), trypsinEDTA, L-glutamine, and antibiotics (Penicillin/Streptomycin) were purchased from Life Technologies (Carlsbad, CA, USA). Endothelial basal medium (EBM-2) and endothelial 
growth BulletKit (EGM-2) were purchased from Lonza. Live/dead ${ }^{\circledR}$ Viability/Cytotoxicity Kit, PrestoBlue ${ }^{\circledR}$ Cell Viability Reagent, Alexa Fluor ${ }^{\circledR}$ 594-Phalloidin and 4',6-diamidino-2phenylindole (DAPI) were purchased from Life Technologies. Rabbit anti-human CD31, mouse anti- $\alpha$-smooth muscle actin ( $\alpha$-SMA) antibodies, Alexa Fluor ${ }^{\circledR}$ 594- or 488-conjugated goat anti-rabbit or goat anti-mouse secondary antibodies were purchased from Abcam (Cambridge, MA, USA). Other chemicals were purchased from Sigma-Aldrich unless otherwise noted.

\subsection{Preparation of Blend Bioink}

GelMA with moderate degree of methacryloyl modification was prepared using the method modified from our previous work [46]. Various concentrations (w/v) of GelMA (5 and $7 \%$ ), alginate $(1,2$, and 3\%) and 4-arm PEGTA $(1,2$, and 3\%), were dissolved in deionized water containing 10\% (v/v) FBS and $25 \mathrm{mM}$ HEPES. In addition, 0.25\% (w/v) photoinitiator was added for inducing photocrosslinking. $\mathrm{CaCl}_{2}$ was also dissolved in $10 \%$ (v/v) FBS solution at $0.3 \mathrm{M}$ concentration as the ionic crosslinking agent. As a chelator for alginate, a solution of 20-mM EDTA in PBS was prepared and stored at $4{ }^{\circ} \mathrm{C}$ until use. All solutions were sterilized and filtered by a $0.22-\mu \mathrm{m}$ filter and stored in an incubator at $37{ }^{\circ} \mathrm{C}$ before use.

\subsection{Printing Setup}

A coaxial nozzle device containing two injection channels was fabricated using different sizes of needles (BD Biosciences, San Jose, CA, USA), which were concentrically fixed by attaching each other using epoxy resin (Devcon, MA, USA). Different sizes of needles ranging from 14 to $30 \mathrm{G}$ were used for fabrication of different size of the perfusable tubes. A commercial bioprinter (Novogen MMX BioprinterTM, Organovo, San Diego, CA, USA) with motor-driven X-Y-Z moving system was used to assemble the nozzle device and print 3D structure of hydrogel bioink by computer-assisted software. The moving speed of the nozzle device ranged from 2 to $6 \mathrm{~mm} \mathrm{~s}^{-1}$. The external channels were connected with $0.3 \mathrm{M} \mathrm{CaCl}_{2}$ solution and the internal channel was connected with bioink, both of which were controlled with microfluidic syringe pumps (Harvard Apparatus) at the extrusion speed of 70 and $35 \mu \mathrm{L}$ $\min ^{-1}$, respectively. The spray of $\mathrm{CaCl}_{2}$ solution was used for ionic crosslinking of the outer surface of printed perfusable tubes when printing. For bioprinting of cell-laden ink, the cells 
were mixed with pre-polymer ink just before use. Immediately after bioprinting, the prepared constructs were exposed to UV light $\left(360-480 \mathrm{~nm}, 6.9-\mathrm{mW} \mathrm{cm}^{-2}\right.$ power, and 8-cm distance from the light source) at designed different time points $(20 \mathrm{~s}, 30 \mathrm{~s}$, and $40 \mathrm{~s})$ for covalently crosslinking, followed by immersion in 20-mM EDTA solution for 5 min for removal of alginate.

\subsection{Bioink Optimization}

\subsubsection{Printability}

The bioink polymer composed of a series of different concentrations (w/v) of components, including GelMA (5 and 7\%), sodium alginate (1, 2, and 3\%) and 4-arm PEGTA (1, 2, and $3 \%$ ), were dissolved in water-FBS-HEPES, respectively. Their printability for creating continuous, complete, uniform, and perfusable tubes with a three-layer structure was tested using the printing device.

\subsubsection{Rheological measurements}

Similarly, the rheological properties of bioink mixture with different ratios of components were analyzed with a rotational rheometer (Anton Paar RHEOPLUS-32, plate-cone geometry). Solutions were kept under mild stirring at $20{ }^{\circ} \mathrm{C}$ for $\sim 2 \mathrm{~h}$ before data acquisition. The test was performed in a closed chamber at $20{ }^{\circ} \mathrm{C}$ to prevent evaporation, where the temperature was consistent with that during the bioprinting process. Each sample was tested in a shear ramp test running from $1 \mathrm{~s}^{-1}$ to $1000 \mathrm{~s}^{-1}$ shear rate over the course of $2 \mathrm{~min}$. Two loading cycles, with $10 \mathrm{~min}$ intervals, were performed before acquiring the rheological data.

\subsubsection{Mechanical properties:}

Mechanical tests were performed for comparison of bioink with different concentrations (w/v) of GelMA (5 and 7\%), sodium alginate (1, 2, and $3 \%$ ) and 4-arm PEGTA (1, 2, and 3\%) dissolved in water-FBS-HEPES, respectively. In brief, the pre-polymer bioink with different ratio of component was pipetted to a prepared cylindrical polydimethylsiloxane (PDMS) mold ( $8 \mathrm{~mm}$ in diameter and $2 \mathrm{~mm}$ in height) and soaked in $0.3 \mathrm{M} \mathrm{CaCl}_{2}$ solution for $2 \mathrm{~min}$, followed by exposing to $6.9 \mathrm{~mW} \mathrm{~cm}^{-2} \mathrm{UV}$ light for 20, 30 and $40 \mathrm{~s}$. The samples were washed with EDTA solution for 5 min after detaching from the mold and incubated in PBS at room temperature for $2 \mathrm{~h}$ to reach the swelling equilibrium. Next, the compressive strength of the samples was tested at a cross speed of $30 \mathrm{~mm} \mathrm{~s}^{-1}$ and a $60 \%$ strain level 
according to previously described procedures using a mechanical testing machine (Instron Model 5542, Norwood, MA, USA) at $37{ }^{\circ} \mathrm{C}$. The compression (mm) and load (N) were then recorded using Bluehill software and the compressive modulus was determined as the slope of the linear region corresponding with $0-10 \%$ strain.

\subsection{Characterization of Printed Constructs}

For clear observation of the structure of the printed constructs, fluorescent beads were mixed with the pre-polymer bioink and printed as the method described above. Different sizes of needles were used for fabrication of perfusable tube with different diameter sizes. Using custom-coded programs, one single perfusable tube was also printed continuously to produce constructs with different geometric shapes. After then, a perfusion test was performed by pumping dyes mixed in cell culture media into the printed perfusable tube using a syringe pump. The final constructs were examined using an inverted fluorescence microscope (Zeiss Axio observer D1, Thornwood, New York, USA) and a laser scanning confocal microscope (Leica SP5 X MP, Wetzlar, 35578 Germany). Moreover, 3D constructs with different layers in a longitudinal plane and different spacing size among adjacent fibers in a horizontal plane were printed for comparison and to evaluate the reliability of printing technology used in this study for precise control of the structure parameters.

Dye-conjugated alginate was prepared by reacting alginate with sulforhodamine 101 cadaverine (Tenova Pharmaceuticals, San Diego, CA, USA) via the EDC/NHS chemistry. Briefly, alginate $(100 \mathrm{mg})$ was dissolved in $5 \mathrm{~mL}$ PBS, followed by the addition of sulforhodamine 101 cadaverine (1 mg), 1-ethyl-3-(3-dimethylaminopropyl)-carbodiimide) (EDC, $3 \mathrm{mg}$ ), and N-hydroxysuccinimide (NHS, $2 \mathrm{mg}$ ). The reaction mixture was stirred at room temperature under protection from light for overnight. The product was purified by dialysis against DI water (3.5-kDa MWCO, Spectrum Labs, Springfield, MA, USA) followed by lyophilizing to afford a violet solid. It should be noted that the degree of fluorescent dye modification of alginate was very low $(<0.5 \%$ of carboxylate groups). Therefore, this rhodamine-conjugated alginate showed similar gelation compared to pristine alginate at the same concentration of $\mathrm{CaCl}_{2}$. The alginate product was used for preparation of blend bioink for printing constructs, and it was then immersed in 20-mM EDTA solution for different time 
points. The fluorescence intensity of the dye-conjugated alginate was determined using fluorescence microscope.

For determination of degradation rate of the printed constructs, the prepared samples with 5 layers $(8 \mathrm{~mm}(\mathrm{~L}) \times 9 \mathrm{~mm}(\mathrm{~W}) \times 4 \mathrm{~mm}(\mathrm{H}))$ were soaked in plate wells with $1 \mathrm{~mL}$ of cell culture media at $37^{\circ} \mathrm{C}$. After designed time points (days $0,4,7$, and 14), the samples were lyophilized and the weight was recorded. The percentage of degradation was calculated by the dry weight after different time points (days 4, 7, and 14) divided by the initial constructs weight (day 0). Moreover, the morphology of the printed constructs was also measured using scanning electron microscope (SEM). The samples were dehydrated using gradient increasing concentrations of ethanol ranging from $50 \%$ to $100 \%(\mathrm{v} / \mathrm{v})$ and freezedried. Then the lyophilized constructs were coated with Au for SEM imagining using a FEI/Philips XL30 FEG ESEM (FEI Co., Eindhoven, The Netherlands) at $15 \mathrm{kV}$.

\subsection{Bioprinting of Cell-encapsulated Constructs}

\subsubsection{Cell Culture, Encapsulation and Bioprinting}

Human umbilical vein endothelial cells (HUVECs) and human mesenchymal stem cells (MSCs) were purchased from American Type Culture Collection (ATCC) (Manassas, VA, USA) and cultured in $\alpha$-MEM containing $10 \%$ FBS, $10 \mathrm{ng} / \mathrm{mL}$ TGF- $\beta 1,1 \%$ glutamine, and 1\% penicillin-streptomycin, and EBM-2 supplemented with EGM-2 BulletKit and 10\% FBS, respectively. All cell lines were cultured in $5 \% \mathrm{CO}_{2}$ at $37{ }^{\circ} \mathrm{C}$ in tissue culture polystyrene flasks. Cell culture medium was replaced every 3 days and cells were passaged when they reached $80-90 \%$ confluency and passages 4-6 were chosen for the subsequent experiments. Based on our previous research [47], mixture of MSCs/HUVECs (1:1) and EBM-2/ $\alpha-M E M$ mixture medium (1:1) were used in this study. Therefore, $3 \times 10^{6}$ cells $\mathrm{mL}^{-1}$ of HUVECs and $3 \times 10^{6}$ cells $\mathrm{mL}^{-1}$ of MSCs were mixed and suspended in $1 \mathrm{~mL}$ of prepolymer solution for bioprinting of vascular constructs with 3 layers $(8 \mathrm{~mm}(\mathrm{~L}) \times 9 \mathrm{~mm}(\mathrm{~W}) \times 2.5 \mathrm{~mm}(\mathrm{H}))$. Immediately after UV exposure (different time of 20, 30, and 40s were used for comparison), the prepared cells-encapsulated constructs were washed with mixture medium for 3 times to remove the excess $\mathrm{CaCl}_{2}$ and photoinitiator, followed by soaking in EDTA solution for 5 min to remove the alginate from the constructs, before culturing in mixture cell media inside the incubator for designed different time points. 


\subsubsection{Cell Viability and Proliferation Assay}

A LIVE/DEAD ${ }^{\circledR}$ Cell Viability Kit was used for assessment of cell viability according to the manufacturer's instructions at day 1, 3 and 7 after bioprinting. The samples were stained with calcein AM (green) and ethidium homodimer-1 (red), followed by observing under an inverted fluorescence microscope (Zeiss Axio Observer D1). At least six images from different areas of three bioprinted constructs for each condition were chosen randomly to counting the number of live and dead cells using NIH ImageJ software, and the cell viability was then expressed as the percentage of the number of live cells to total cells number. Meanwhile, cell metabolic activity was quantified using a $10 \%(\mathrm{v} / \mathrm{v})$ PrestoBlue $^{\circledR}$ Cell Viability Reagent in $\alpha$-MEM without phenol red at day $0,1,3,7$ and 14 after bioprinting. The samples were washed after removal of the media and added with PrestoBlue solution, followed by incubation at $37{ }^{\circ} \mathrm{C}$ for $2 \mathrm{~h}$. Then $200 \mu \mathrm{L}$ of the supernatant was collected respectively from at least six parallel samples for fluorescence measurement with plate reader (Synergy HT, Biotek). The printed constructs without cells was used as control. The OD values were normalized against that of day 0 .

\subsubsection{Imaging and Analysis:}

For observation of the cells morphology and functionality with culture time, immunofluorescence staining of F-actin, CD31, $\alpha$-SMA, and nuclei was performed for the printed constructs after designed time points of culture. In brief, samples were fixed in $4 \%$ (w/v) paraformaldehyde for $15 \mathrm{~min}$, and then soaked in $0.1 \%(\mathrm{v} / \mathrm{v})$ Triton X-100 in PBS for 30 min to permeabilize the cell membrane, followed by blocking with $1 \%$ BSA in PBS for 1 $\mathrm{h}$ at room temperature. For F-actin cytoskeleton staining, the samples were then soaked in 1:40 dilution of Alexa Fluor 594-phalloidin in 0.1\% BSA for 45 min at $37{ }^{\circ} \mathrm{C}$. For CD31 and $\alpha$-SMA staining, the samples were then incubated with primary antibodies, mouse antihuman CD31 (1:50) and rabbit anti-human $\alpha$-SMA (1:100), both for overnight at $4^{\circ} \mathrm{C}$. After washing with PBS, the samples were incubated with Alexa Fluor 594-goat anti-mouse and Alexa Fluor 488 goat anti-rabbit secondary antibody $(1: 200)$ for $1 \mathrm{~h}$ at $37{ }^{\circ} \mathrm{C}$. Finally, the nuclei were stained with DAPI $(1: 1000)$ for 5 min at $37^{\circ} \mathrm{C}$. All antibodies were diluted with $0.1 \%$ BSA. The final samples were examined using an inverted laser scanning confocal microscope. 


\subsection{Statistical Analysis}

Statistical analysis was performed using Statistical Package for the Social Sciences, Version 11.0 for Windows (SigmaStat, SPSS Science, Chicago, IL, USA). Data were collected from at least six parallel samples. The results were reported as the mean \pm standard deviation (SD). Values were considered to be significant at $\mathrm{p}<0.05$.

\section{Results and Discussion}

\subsection{The Bioprinting Setup and Bioink Properties}

The schematic diagrams in Fig. 1A and $\mathbf{B}$ show the bioprinting procedure to create highly organized perfusable vascular structures using a simple, one-step 3D bioprinting technique based on a multilayered coaxial nozzle device in combination with cell-laden blend bioink. The fabrication mechanism is based on two independent crosslinking processes for our specially designed blend bioink composed of GelMA, alginate, and 4-arm PEGTA [44]. Ionic crosslinking of the alginate component is first induced by complexation of alginate in the bioink delivered through the sheath layer with $\mathrm{Ca}^{2+}$ delivered through both the core channel of the printhead and the ambient spray of $\mathrm{CaCl}_{2}$ solution, providing temporary structural stability during the bioprinting process. Covalent photocrosslinking of the GelMA in the bioink is then subsequently induced by exposing the bioprinted constructs to light, resulting in permanent fixation of its architecture. This process is cytocompatible, allowing convenient encapsulation of endothelial and associated cells during the bioprinting process. The alginate component, which is bio-inert, can then be dissolved using a $\mathrm{Ca}^{2+}$-chelating agent such as 20$\mathrm{mM}$ EDTA to render improved spreading and proliferation of the cells in the bioprinted construct [41], eventually forming a biomimetic vasculature. However, it was expected that the photocrosslinked construct after removing alginate possessed low mechanical strength, which would limit the stability of multilayered construct. To solve this problem, 4-arm PEGTA was chosen to enhance mechanical property and stabilize the crosslinked matrix. Moreover, the increased branching of PEG molecules could further result in an improvement in the biomolecule diffusivity and pore size that would support better cell growth than linear PEG [48]. Multilayered coaxial nozzles consisting of two or three needles assembled concentrically were successfully fabricated for direct extrusion of perfusable tubes with 
consistent or varying diameters (Fig. 1C I and II). When using tri-layered nozzles, the bioink was delivered through the second and third sheaths, while $\mathrm{CaCl}_{2}$ solution was simultaneously delivered through both core channel and ambient spray. The hollow tube with varying diameter could be achieved by alternately switching on the outermost bioink channel on demand (Fig. 1C II). Therefore, the integration of this novel nozzle system with the Organovo Novogen MMX Bioprinter ${ }^{\mathrm{TM}}$ further allows for convenient deposition of the perfusable tube in three dimensions.

Evaluation of the printability of blend bioink of different formulations using the designed coaxial nozzles revealed that printing feasibility was achieved with bioink containing 2 or $3 \%$ (w/v) alginate, 5 or $7 \%$ (w/v) GelMA, and 2 or 3\% (w/v) 4-arm PEGTA. As displayed in Fig. $\mathbf{2 A}$, the printability of the blend bioink was achieved by increasing the concentration of alginate and 4-arm PEGTA. Based on the printability results, we believe that the high concentration of GelMA up to $7 \%$ would be the optimal condition for cell responses considering the fact that GelMA has intrinsic cell-adhesion moieties [38]. We preferred a high concentration of alginate (3\%) in order to improve printability of bioink during printing process. In addition, 2\% 4-arm PEGTA was deemed maximum concentration that could be used due to the significantly increased viscosity of the blend bioink. To confirm the viscosity of the blend bioink, rheological properties of GelMA, alginate, 4-arm PEGTA, and their mixtures were measured (Fig. 2B). Interestingly, it was observed that by adding alginate to GelMA solutions, viscosity of the resultant GelMA/alginate mixture solutions became much higher than that of pure GelMA or alginate solutions across the entire range of shear rates tested (1-1000 $\mathrm{s}^{-1}$, Fig. 2B), thus facilitating the bioprinting process with good fidelity due to the significantly higher viscosity when the shear stress is released. The significant increase in viscosity of GelMA/alginate mixture solutions could be partly attributed to the electrostatic interactions between positively charged GelMA derived from type-A gelatin and negatively charged alginate [49]. Meanwhile, the addition of 4-arm PEGTA increased viscosity of the blend bioink due to the increased mass concentration. At $20{ }^{\circ} \mathrm{C}$, the viscosity of the bioinks increased from 28 to $54 \mathrm{~Pa}$ s with elevated concentrations of 4-arm PEGTA from 0 to 3\%, at a shear rate of $7.74 \mathrm{~s}^{-1}$ present during the extrusion process, which is comparable to those reported in the literature [50]. Moreover, viscosity of the tri-component bioink was also 
found to decrease with increasing shear rate, suggesting a shear-thinning behavior, which allowed convenient extrusion of the blend bioink from the nozzles and adjustment of the deposition speed without causing clogging issue and an improvement in the resolution of the printing process. Based on these systematic analyses regarding the properties of our specially designed bioink [44, 51], we decided to use a mixture composed of 7\% GelMA, 3\% alginate, and $2 \% 4$-arm PEGTA as the bioink for subsequent experiments.

To confirm the effect of 4-arm PEGTA to improve mechanical properties of GelMA hydrogel, unconfined compression tests were performed with acellular bulk structures swollen in PBS at room temperature. We found that the photocrosslinked construct after removing alginate possessed low mechanical strength $(13.2 \pm 1.2 \mathrm{kPa}$, Fig. 2C), which limited the stability of multiple-layered construct. However, the compressive moduli of the hydrogel containing $1 \%, 2 \%$, and 3\% 4-arm PEGTA $(24.2 \pm 1.2 \mathrm{kPa}, 34.5 \pm 4.4 \mathrm{kPa}, 50.7 \pm$ $3.0 \mathrm{kPa}$, respectively) were all significantly higher than that of the hydrogel without 4-arm PEGTA $(13.2 \pm 1.2 \mathrm{kPa})$ when exposed to $6.9 \mathrm{~mW} / \mathrm{cm}^{2} \mathrm{UV}$ for $30 \mathrm{~s}$. Enhanced mechanical properties of the GelMA/4-arm PEGTA hydrogels ensures the stability of the bioprinted perfusable constructs by providing sufficient strength to withstand internal and external mechanical loads.

\subsection{Fabrication and Characterization of Bioprinted Perfusable tube Constructs}

The designed coaxial nozzles allowed us to smoothly deposit the optimized bioink in the form of hollow tubes with transverse dimensions, which could be achieved by adjusting the sizes of the external and internal needles (Fig. 3A). By combining different sizes of the internal needles (ranging from $27 \mathrm{G}$ to $30 \mathrm{G}$ ) and the external needles (ranging from $18 \mathrm{G}$ to $25 \mathrm{G}$ ), series of tubes were printed with different sizes ( Fig. 3A I). A wide range of tube diameter could be achieved (Fig. 3A II), with average values from approximately $500 \mu \mathrm{m}$ to $1500 \mu \mathrm{m}$ for the outer diameter, $400 \mu \mathrm{m}$ to $1000 \mu \mathrm{m}$ for the inner diameter, and $60 \mu \mathrm{m}$ to 280 $\mu \mathrm{m}$ for wall thickness, respectively, covering much wider range of potential applications compared with previous reports in which only limited sizes of hollow tubes could be created [27-30]. Similarly, the fixed size of external needle (20G) coupled with different sizes of the internal needles (ranging from $25 \mathrm{G}$ to $30 \mathrm{G}$ ) could also generate perfusable tubes with 
different inner diameters and wall thicknesses (Fig. 3A III). In addition, studies have shown that large wall thicknesses of engineered vessels may block the transport of oxygen and nutrients, while very small wall thicknesses would lead to limited volume for the encapsulated cells to align and spread besides the insufficient mechanical strength [26, 30]. For these reasons, the nozzle made of a $20 \mathrm{G}$ external needle and a $30 \mathrm{G}$ internal needle was chosen for subsequent experiments to extrude perfusable tubes with an outer diameter of 800 $\mu \mathrm{m}$ and a wall thickness of $110 \mu \mathrm{m}$, which were expected to meet both requirements for allowing sufficient nutrients diffusion and supporting cell growth [30, 52].

By programming the 3D bioprinter, single hollow tubes could be continuously extruded and smoothly deposited to create perfusable networks with desired shapes and architecture (Fig. 3B). The printed structures maintained high fidelity without bioink accumulation even at corners in the angular geometries where the printing directions were sharply altered. This feature has set up the basis for future application of this innovative technology to generate vascular structures containing highly curvy tortuosity to mimic the shapes of the blood vessels present in the human body. More importantly, it was further demonstrated that, the diameter of a single tube could be modulated on demand by simply switching on/off the bioink flow in the outmost channel of the printhead (Fig. 1C and Fig. 3C), or by changing the flow rates of the bioink extrusion and/or moving speed of the printhead. The bioprinted fibers with varying diameters maintained their hollow structures and could be perfused along the entire lengths (Fig. 3C II and IV), closely mimicking the hierarchical size distribution of the vasculature connecting different anatomical sites in vivo. This unique capability of our bioprinting system provides unprecedented convenience in fabricating hollows tubes consisting of segments of continuously changing or multiple sizes when necessary, without the need of replacing the nozzles. Such degree of flexibility in simultaneously tuning the parameters of the bioprinted fibrous structures while maintaining the hollow lumens is hardly achievable with recently reported techniques [27-30].

Our bioprinting technique also enables precise control over the architecture of the printed constructs to acquire different degrees of anisotropy and produce stacked constructs. As shown in Fig. 4A I and Movie S1, we were able to fabricate 3D constructs with different spacing between adjacent deposited perfusable fibers, including $2 \times 2,2 \times 3$, and $3 \times 3$ in the 
aspect ratios of the unit grid. Constructs with different numbers of layers (e.g. 2, 5, and 10 layers) could also be achieved (Fig. 4A II). These different structures of the bioprinted constructs may be utilized to mimic various types of native tissues by supporting different alignment needs. Furthermore, successful preparation of 3D multilayered constructs (10 layers with a size of $8 \mathrm{~mm}(\mathrm{~L}) \times 9 \mathrm{~mm}(\mathrm{~W}) \times 7 \mathrm{~mm}(\mathrm{H})$, Fig. S1) confirmed the robustness of the designed bioprinting system and indicated a great potential to develop larger vascularized tissue constructs. In Fig. 4B, the confocal images of the cross-section and top-view of printed constructs with 10 layers of stacking perfusable tube further clearly demonstrated a fully interconnected luminal structure without obvious signs of collapse or rupture throughout the entire volume of the bioprinted construct, although the crossing tubes at the sites of stacking showed minor deformation. This was confirmed by successful perfusion of red fluorescent microbeads through the entire length of the hollow tubes (Fig. 4C and Movie S2). The perfusable property of the bioprinted tubes lays down the basis for their use as blood vessel mimics, which should be attributed to the enhanced mechanical strength of the blend bioink by PEGTA integration to support the integrity of the printed hollow structures.

\subsection{Biological Performance of Cell-laden Constructs}

To improve the spreading, migration, and proliferation of the cells in the bioprinted construct, ionically crosslinked alginate was then dissolved by EDTA. To confirm the removal of alginate, we printed acellular structures using a fluorescent-labeled alginate to make bioink and took fluorescent pictures over a time period (Fig. S2A). The fluorescent micrographs of the bioprinted constructs clearly showed that most of fluorescently labeled alginate was removed from the constructs after 5 min of EDTA washing, while the constructs could still maintain their intact structures due to the presence of 4-arm PEGTA. Scanning electron microscopy (SEM) images of the interior of a construct also revealed higher porosity of the blend hydrogel comprising of the tube wall after removal of the alginate (Fig. S2B and S2C), which would potentially facilitate cell migration and spreading [41], inducing the formation of highly organized, intact vessels. Importantly, the density of encapsulated cell in the hollow microfibers did not significantly decrease after removal of alginate (Fig. S2D and S2E). All of these characterization results have demonstrated the favorable physiochemical 
properties of our optimized bioink for printing complex 3D perfusable constructs, as well as reliability and flexibility of our bioprinting technology for fabricating a wide variety of $2 \mathrm{D}$ and $3 \mathrm{D}$ patterns of interest.

We further encapsulated vascular cells in the bioink to demonstrate the possibility to directly bioprint biologically relevant vascular constructs. In particular, co-culture of HUVECs and MSCs was used in this study to better mimic the natural blood vessels composed of multiple cell types. Our pervious study has also shown that co-culture of HUVECs and MSCs significantly promoted the proliferation of HUVECs compared to monoculture $[11,47]$. It might be a result of interactions between the two types of cells via direct cell-cell contacts and diffusible paracrine signaling, which was further confirmed in the present study that the spreading and morphology of co-culture of HUVECs and MSCs were superior to monoculture of HUVECs (Fig. S3). It is well established that MSCs can release bioactive growth factors such as vascular endothelial growth factor (VEGF) and thus promote functions of HUVECs [53, 54]. More importantly, it is also well known that MSCs have the ability to proliferate in vitro and differentiate into a series of mesoderm-type lineages, including osteoblasts, chondrocytes, adipocytes, myocytes, as well as vascular cells, depending on the culture conditions $[55,56]$. The most potent inducer of differentiation from MSCs to vascular smooth muscle cells (SMCs) is TGF- $\beta 1$ treatment [55, 57], which therefore was added into the EBM-2/ $\alpha$-MEM mixture medium (1:1) for inducing SMCs differentiation of MSCs in the present study.

For clear observation of the spreading and proliferation of the encapsulated cells, constructs composed of 3 layers with a size of $8 \mathrm{~mm}(\mathrm{~L}) \times 9 \mathrm{~mm}(\mathrm{~W}) \times 2.5 \mathrm{~mm}(\mathrm{H})$ were bioprinted. The fluorescence micrographs (Fig. 5 and Fig. S4) revealed that the cells were homogeneously distributed within the bioprinted constructs and meanwhile the constructs could maintain their intact structures. The cell viability was then compared for bioprinted constructs fabricated by exposure to different UV crosslinking times $(20,30$, and $40 \mathrm{~s})$. The results showed that the percentages of viable cells within the bioprinted constructs at UV exposures of $20 \mathrm{~s}$ and $30 \mathrm{~s}$ exceeded $80 \%$ after 1, 3, and 7 days of culture, significantly higher than those exposed to UV for $40 \mathrm{~s}$ (Fig. 5A, B and Fig. S4). This result is consistent with our previous finding that UV exposure times of below $30 \mathrm{~s}$ for bioprinted tissue 
constructs do not affect the viability of the encapsulated cells [44]. To verify this assumption, cell proliferation assay was further performed after bioprinting (Fig. 5C). It was found out that cellular metabolic activity within the bioprinted vascular constructs increased gradually as a function of culture time, which was particularly expedited at 7 and 14 days of culture. This further confirmed that the used bioink composition was cytocompatible and promoted cellular growth overtime. Moreover, at both time points the metabolic activities of the samples exposed to UV for $20 \mathrm{~s}$ and $30 \mathrm{~s}$ were significantly higher those undergoing $40 \mathrm{~s}$ of crosslinking, suggesting limited cell proliferation when the crosslinking density/matrix stiffness was increased, which was consistent with other reports that the mechanical properties of the scaffold affect cell behavior such as proliferation, spreading, and migration $[58,59]$. Our bioprinting approach for direct deposition of perfusable vascular structures revealed a similar if not higher, cell viability, compared to reports by other studies using the same extrusion-based bioprinting method [10, 30, 60, 61].

F-actin/DAPI staining was further performed to monitor the spreading and morphology of the encapsulated cells within the bioprinted constructs (Fig. 5D; $30 \mathrm{~s} \mathrm{UV} \mathrm{crosslinking).} \mathrm{As}$ expected, no noticeable cell spreading was found on Day 1 and all of cells still maintained rounded shapes. Starting from Day 7 through Day 21, the cells gradually spread out initially from spindle shapes to later on long stripe-like shapes, scattering along the walls of the perfusable fibers across different layers, potentially due to the degradation of the hydrogel. The proliferation of the encapsulated cells was also prominent, eventually reaching confluence after 21 days of culture to fill the entire volume of the tubular walls and form an integrated vessel-like structure as a fine balance between hydrogel degradation and cell proliferation. The degradation profiles further showed that the bioprinted vascular constructs gradually degraded as a function of incubation time in the culture medium (Fig. 5E). The degradation rate of scaffolds at $40 \mathrm{~s}$ of UV exposure was slower than those with $20 \mathrm{~s}$ and $30 \mathrm{~s}$ crosslinking, with more than $60 \%$ of mass remaining for $40 \mathrm{~s}$ of UV exposure time and less than $40 \%$ of mass remaining for samples crosslinked for $20 \mathrm{~s}$ and $30 \mathrm{~s}$, after 14 days of analysis. This result suggests that longer UV crosslinking time rendered the hydrogel network stiffer and therefore decreased the degradation rate. This rate was also slower than those of pure gelatin hydrogels or other hybrid hydrogels based on GelMA [51, 62], potentially due to 
the addition of 4-arm PEGTA that enhanced the crosslinking density of the bioink. This observation is in line with other reports where inclusion of 4-arm PEGTA decreased the degradation rate of blend protein-based hydrogel [63]. The moderate degradation rate had made our bioink more suitable for printing 3D vascular constructs for longer term applications, by facilitating the cellular responses and formation of neotissues. It was also found that different UV crosslink times exerted different effects on the spreading and morphology of the encapsulated cells (Fig. S5). The results indicated that, the constructs exposed to UV for $30 \mathrm{~s}$ displayed the best cell spreading and organization compared to those crosslinked for $20 \mathrm{~s}$ and $40 \mathrm{~s}$. This is because stiffer hydrogel network resulted from prolonged UV exposure (>30 s) hindered the proliferation, spreading, and potentially migration of the cells. In contrast, inadequate crosslinking of hydrogel resulted from shorter exposure time $(20 \mathrm{~s})$ caused insufficient mechanical strength and thus less intact structure for encapsulated cells to attach and spread.

The confocal micrographs in Fig. 6A reveal the multilayered nature of the fully vascularized tubes and the hollow lumen structure, closely mimicking the important aspects of the native blood vessels. Excellent spreading and proliferation of the cells within the bioprinted vascular constructs indicated the great potential for bioprinting complex tissue constructs with built-in vascularization potential. We further demonstrated that the coencapsulated HUVECs and MSCs inside the perfusable fibers could mature into functional cell types. Immunofluorescence staining for biomarkers including CD31 and $\alpha$-SMA was performed. CD31 is an endothelial-specific adhesion protein expressed on the membranes of endothelial cells and comprises a major chunk of the intercellular junction complexes [64, 65], while $\alpha$-SMA is the actin isoform that predominates within vascular SMCs and plays an important role in angiogenesis [66, 67]. As revealed in Fig. 6B, both markers could be clearly observed after 14 and 21 days of culture, showing the expressions of CD31 at the interfaces of HUVECs and $\alpha$-SMA for SMCs derived from MSCs, which are crucial for vessel formation and stabilization. The expression of both markers became more pronounced overtime along with cell spreading and proliferation, indicative of early-stage vessel maturation resembling the native vasculature. These results demonstrated that our optimized bioink could provide a favorable biochemical and physical microenvironment for the 
encapsulated vascular cells to remodel inside the perfusable vascular fibers, facilitating the ultimate formation of functional vascular structures. However, it was found that the compressive moduli of the constructs significantly decreased after 21 days of culture mainly due to the degradation of the GelMA component (Fig. S6). It is therefore difficult to maintain perfusability using the current bioinks after 21 days of culture. In addition, the thin walls of the constructs resulted in mechanical instability making the measurement of other mechanical properties such as burst pressure difficult in this study. However, we are now in the process of designing better bioink formulations that may potentially solve such an issue, which will be presented in future reports.

\section{Conclusions}

In this study, we presented a convenient and versatile 3D bioprinting strategy for direct deposition of cell-laden perfusable vascular constructs. A novel blend ink consisted of GelMA, alginate, and 4-arm PEGTA was developed with favorable physicochemical characteristics that supported the proliferation and early maturation of vascular cells as well as bioprinting of complex 3D vasculature. Moreover, a multilayered coaxial nozzle containing concentric channels was designed for continuous generation of perfusable constructs with multiple layers and various diameters in a single-step process. Further combination with vascular and associated cell types encapsulated in the bioink could induce the formation of viable and functional vessels resembling early maturation of the native vasculature. Clearly possessing distinct advantages over conventional complicated microfabrication and sacrificial templating approaches (i.e. templates are removed from hydrogels to generate hollow channels), we believe that our novel single-step strategy holds great potential for applications in engineering pre-vascularized tissue constructs.

\section{Acknowledgements}

This paper is sponsored by the Institute for Soldier Nanotechnology, National Institutes of Health (HL092836, EB02597, AR057837, HL099073), the National Science Foundation (DMR0847287), the Office of Naval Research Young Investigator award, ONR PECASE Award, a Discovery grant from National Sciences and Engineering Research Council of 
Canada and Scientific and Technological Research Council of Turkey (TUBITAK). P.S.G.O. acknowledges funding (B.14.2.TBT.0.06.01-219-115543) from the Scientific and Technological Research Council of Turkey (TUBITAK). Y.S.Z. acknowledges the National Cancer Institute of the National Institutes of Health K99/R00 Pathway to Independence Award (1K99CA201603-01A1). S.R.S. would like to recognize and thank Brigham and Women's Hospital President Betsy Nabel, MD, and the Reny family, for the Stepping Strong Innovator Award through their generous funding. The authors would like to thank Reginald Avery for rheology analysis. The authors declare no conflict of interests in this work.

\section{References}

[1] Dvir T, Timko BP, Kohane DS, Langer R. Nanotechnological strategies for engineering complex tissues. Nature Nanotechnology. 2011;6:13-22.

[2] Lutolf MP, Gilbert PM, Blau HM. Designing materials to direct stem-cell fate. Nature. 2009;462:433-41.

[3] Engelmayr GC, Jr., Cheng M, Bettinger CJ, Borenstein JT, Langer R, Freed LE. Accordion-like honeycombs for tissue engineering of cardiac anisotropy. Nature materials. 2008;7:1003-10.

[4] Eschenhagen T, Eder A, Vollert I, Hansen A. Physiological aspects of cardiac tissue engineering. American journal of physiology Heart and circulatory physiology. 2012;303:H133-43.

[5] Zhang YS, Xia Y. Multiple facets for extracellular matrix mimicking in regenerative medicine. Nanomedicine. 2015;10:689-92.

[6] Freed LE, Jr. GCE, Borenstein JT, Moutos FT, Guilak F. Advanced Material Strategies for Tissue Engineering Scaffolds. Adv Mater. 2009;21:3410-8.

[7] Leijten J, Rouwkema J, Zhang YS, Nasajpour A, Dokmeci MR, Khademhosseini A. Advancing Tissue Engineering: a Tale of Nano, Micro and Macro Scale Integration. Small. 2016;10:2130-45.

[8] Moon JJ, West JL. Vascularization of Engineered Tissues: Approaches to Promote Angiogenesis in Biomaterials. Curr Top Med Chem. 2008;8:300-10.

[9] Bae H, Puranik AS, Gauvin R, Edalat F, Carrillo-Conde B, Peppas NA, et al. Building Vascular Networks. Sci Transl Med. 2012;4:160ps23-ps23.

[10] Kolesky DB, Truby RL, Gladman AS, Busbee TA, Homan KA, Lewis JA. 3D bioprinting of vascularized, heterogeneous cell-laden tissue constructs. Advanced materials. 2014;26:3124-30.

[11] Chen YC, Lin RZ, Qi H, Yang Y, Bae H, Melero-Martin JM, et al. Functional Human Vascular Network Generated in Photocrosslinkable Gelatin Methacrylate Hydrogels. Advanced functional materials. 2012;22:2027-39.

[12] Asakawa N, Shimizu T, Tsuda Y, Sekiya S, Sasagawa T, Yamato M, et al. Pre-vascularization of in vitro three-dimensional tissues created by cell sheet engineering. Biomaterials. 2010;31:3903-9.

[13] Hasan A, Paul A, Vrana NE, Zhao X, Memic A, Hwang YS, et al. Microfluidic techniques for development of 3D vascularized tissue. Biomaterials. 2014;35:7308-25.

[14] Lin RZ, Melero-Martin JM. Bioengineering human microvascular networks in immunodeficient mice. Journal of visualized experiments : JoVE. 2011:e3065.

[15] Murphy SV, Atala A. 3D bioprinting of tissues and organs. Nature biotechnology. 2014;32:773-85.

[16] Wüst S, Müller R, Hofmann S. Controlled positioning of cells in biomaterials_-approaches towards 3D 
tissue printing. Journal of functional biomaterials. 2011;2:119-54.

[17] Melchels FPW, Domingos MAN, Klein TJ, Malda J, Bartolo PJ, Hutmacher DW. Additive manufacturing of tissues and organs. Progress in Polymer Science. 2012;37:1079-104.

[18] Radisic M, Yang L, Boublik J, Cohen RJ, Langer R, Freed LE, et al. Medium perfusion enables engineering of compact and contractile cardiac tissue. American journal of physiology Heart and circulatory physiology. 2004;286:H507-16.

[19] Griffith CK, Miller C, Sainson RC, Calvert JW, Jeon NL, Hughes CC, et al. Diffusion limits of an in vitro thick prevascularized tissue. Tissue engineering. 2005;11:257-66.

[20] Skardal A, Zhang J, Prestwich GD. Bioprinting vessel-like constructs using hyaluronan hydrogels crosslinked with tetrahedral polyethylene glycol tetracrylates. Biomaterials. 2010;31:6173-81.

[21] Tocchio A, Tamplenizza M, Martello F, Gerges I, Rossi E, Argentiere S, et al. Versatile fabrication of vascularizable scaffolds for large tissue engineering in bioreactor. Biomaterials. 2015;45:124-31.

[22] Lee VK, Kim DY, Ngo H, Lee Y, Seo L, Yoo S-S, et al. Creating perfused functional vascular channels using 3D bio-printing technology. Biomaterials. 2014;35:8092-102.

[23] Bertassoni LE, Cecconi M, Manoharan V, Nikkhah M, Hjortnaes J, Cristino AL, et al. Hydrogel bioprinted microchannel networks for vascularization of tissue engineering constructs. Lab Chip. 2014;14:2202-11.

[24] Kolesky DB, Truby RL, Gladman AS, Busbee TA, Homan KA, Lewis JA. 3D Bioprinting of Vascularized, Heterogeneous Cell-Laden Tissue Constructs. Adv Mater. 2014;26:3124-30.

[25] Miller JS, Stevens KR, Yang MT, Baker BM, Nguyen D-HT, Cohen DM, et al. Rapid casting of patterned vascular networks for perfusable engineered three-dimensional tissues. Nat Mater. 2012;11:768-74.

[26] Norotte C, Marga FS, Niklason LE, Forgacs G. Scaffold-free vascular tissue engineering using bioprinting. Biomaterials. 2009;30:5910-7.

[27] Gao Q, He Y, Fu JZ, Liu A, Ma L. Coaxial nozzle-assisted 3D bioprinting with built-in microchannels for nutrients delivery. Biomaterials. 2015;61:203-15.

[28] Zhang Y, Yu Y, Ozbolat IT. Direct bioprinting of vessel-like tubular microfluidic channels. J Nanotechnol Eng Med. 2013;4:020902.

[29] Zhang Y, Yu Y, Akkouch A, Dababneh A, Dolati F, Ozbolat IT. In Vitro Study of Directly Bioprinted Perfusable Vasculature Conduits. Biomaterials science. 2015;3:134-43.

[30] Zhang Y, Yu Y, Chen H, Ozbolat IT. Characterization of printable cellular micro-fluidic channels for tissue engineering. Biofabrication. 2013;5:025004.

[31] Slaughter BV, Khurshid SS, Fisher OZ, Khademhosseini A, Peppas NA. Hydrogels in regenerative medicine. Advanced materials. 2009;21:3307-29.

[32] Khademhosseini A, Langer R. Microengineered hydrogels for tissue engineering. Biomaterials. 2007;28:5087-92.

[33] Burdick JA, Prestwich GD. Hyaluronic acid hydrogels for biomedical applications. Advanced materials. 2011;23:H41-56.

[34] Helary C, Bataille I, Abed A, Illoul C, Anglo A, Louedec L, et al. Concentrated collagen hydrogels as dermal substitutes. Biomaterials. 2010;31:481-90.

[35] Hoffman AS. Hydrogels for biomedical applications. Adv Drug Del Rev. 2012;64:18-23.

[36] Malda J, Visser J, Melchels FP, Jüngst T, Hennink WE, Dhert WJ, et al. 25th anniversary article: engineering hydrogels for biofabrication. Adv Mater. 2013;25:5011-28.

[37] Xing Q, Yates K, Vogt C, Qian Z, Frost MC, Zhao F. Increasing mechanical strength of gelatin hydrogels by divalent metal ion removal. Scientific reports. 2014;4:4706.

[38] Moon JJ, Saik JE, Poche RA, Leslie-Barbick JE, Lee SH, Smith AA, et al. Biomimetic hydrogels with pro- 
angiogenic properties. Biomaterials. 2010;31:3840-7.

[39] Kang HW, Lee SJ, Ko IK, Kengla C, Yoo JJ, Atala A. A 3D bioprinting system to produce human-scale tissue constructs with structural integrity. Nature biotechnology. 2016;34:312-9.

[40] Yue K, Trujillo-de Santiago G, Alvarez MM, Tamayol A, Annabi N, Khademhosseini A. Synthesis, properties, and biomedical applications of gelatin methacryloyl (GelMA) hydrogels. Biomaterials. 2015;73:25471.

[41] Tamayol A, Najafabadi AH, Aliakbarian B, Arab-Tehrany E, Akbari M, Annabi N, et al. Hydrogel Templates for Rapid Manufacturing of Bioactive Fibers and 3D Constructs. Advanced healthcare materials. 2015;4:2146-53.

[42] Akbari M, Tamayol A, Laforte V, Annabi N, Najafabadi AH, Khademhosseini A, et al. Composite Living Fibers for Creating Tissue Constructs Using Textile Techniques. Advanced functional materials. 2014;24:40607.

[43] Morch YA, Donati I, Strand BL, Skjak-Braek G. Effect of $\mathrm{Ca} 2+, \mathrm{Ba} 2+$, and $\mathrm{Sr} 2+$ on alginate microbeads. Biomacromolecules. 2006;7:1471-80.

[44] Colosi C, Shin SR, Manoharan V, Massa S, Costantini M, Barbetta A, et al. Microfluidic Bioprinting of Heterogeneous 3D Tissue Constructs Using Low-Viscosity Bioink. Advanced materials. 2016;28:677-84.

[45] Schukur L, Zorlutuna P, Cha JM, Bae H, Khademhosseini A. Directed differentiation of size-controlled embryoid bodies towards endothelial and cardiac lineages in RGD-modified poly(ethylene glycol) hydrogels. Advanced healthcare materials. 2013;2:195-205.

[46] Nichol JW, Koshy ST, Bae H, Hwang CM, Yamanlar S, Khademhosseini A. Cell-laden microengineered gelatin methacrylate hydrogels. Biomaterials. 2010;31:5536-44.

[47] Kang Y, Kim S, Fahrenholtz M, Khademhosseini A, Yang Y. Osteogenic and angiogenic potentials of monocultured and co-cultured human-bone-marrow-derived mesenchymal stem cells and human-umbilical-vein endothelial cells on three-dimensional porous beta-tricalcium phosphate scaffold. Acta biomaterialia. 2013;9:4906-15.

[48] Lee S, Tong X, Yang F. Effects of the poly(ethylene glycol) hydrogel crosslinking mechanism on protein release. Biomaterials science. 2016;4:405-11.

[49] Wang H, Hansen MB, Lowik DW, van Hest JC, Li Y, Jansen JA, et al. Oppositely charged gelatin nanospheres as building blocks for injectable and biodegradable gels. Advanced materials. 2011;23:H119-24.

[50] Pati F, Jang J, Ha DH, Won Kim S, Rhie JW, Shim JH, et al. Printing three-dimensional tissue analogues with decellularized extracellular matrix bioink. Nature communications. 2014;5:3935.

[51] Hutson CB, Nichol JW, Aubin H, Bae H, Yamanlar S, Al-Haque S, et al. Synthesis and characterization of tunable poly(ethylene glycol): gelatin methacrylate composite hydrogels. Tissue engineering Part A. 2011;17:1713-23.

[52] Choi NW, Cabodi M, Held B, Gleghorn JP, Bonassar LJ, Stroock AD. Microfluidic scaffolds for tissue engineering. Nature materials. 2007;6:908-15.

[53] Mayer H, Bertram H, Lindenmaier W, Korff T, Weber H, Weich H. Vascular endothelial growth factor (VEGF-A) expression in human mesenchymal stem cells: autocrine and paracrine role on osteoblastic and endothelial differentiation. Journal of cellular biochemistry. 2005;95:827-39.

[54] Furumatsu T. Vascular Endothelial Growth Factor Principally Acts as the Main Angiogenic Factor in the Early Stage of Human Osteoblastogenesis. Journal of Biochemistry. 2003;133:633-9.

[55] Vater C, Kasten P, Stiehler M. Culture media for the differentiation of mesenchymal stromal cells. Acta biomaterialia. 2011;7:463-77.

[56] Charbord P. Bone marrow mesenchymal stem cells: historical overview and concepts. Human gene therapy. 
2010;21:1045-56.

[57] Sinha S, Hoofnagle MH, Kingston PA, McCanna ME, Owens GK. Transforming growth factor-beta1 signaling contributes to development of smooth muscle cells from embryonic stem cells. American journal of physiology Cell physiology. 2004;287:C1560-8.

[58] Onoe H, Okitsu T, Itou A, Kato-Negishi M, Gojo R, Kiriya D, et al. Metre-long cell-laden microfibres exhibit tissue morphologies and functions. Nature materials. 2013;12:584-90.

[59] Ehrbar M, Sala A, Lienemann P, Ranga A, Mosiewicz K, Bittermann A, et al. Elucidating the role of matrix stiffness in 3D cell migration and remodeling. Biophysical journal. 2011;100:284-93.

[60] Duan B, Hockaday LA, Kang KH, Butcher JT. 3D bioprinting of heterogeneous aortic valve conduits with alginate/gelatin hydrogels. Journal of biomedical materials research Part A. 2013;101:1255-64.

[61] Ouyang L, Yao R, Chen X, Na J, Sun W. 3D printing of HEK 293FT cell-laden hydrogel into macroporous constructs with high cell viability and normal biological functions. Biofabrication. 2015;7:015010.

[62] Camci-Unal G, Cuttica D, Annabi N, Demarchi D, Khademhosseini A. Synthesis and characterization of hybrid hyaluronic acid-gelatin hydrogels. Biomacromolecules. 2013;14:1085-92.

[63] Rutz AL, Hyland KE, Jakus AE, Burghardt WR, Shah RN. A multimaterial bioink method for 3D printing tunable, cell-compatible hydrogels. Advanced materials. 2015;27:1607-14.

[64] Ayalon O, Sabanai H, Lampugnani M-G, Dejana E, Geiger B. Spatial and temporal relationships between cadherins and PECAM-1 in cell-cell junctions of human endothelial cells. The Journal of cell biology. 1994;126:247-58.

[65] Ilan N, Cheung L, Pinter E, Madri JA. Platelet-endothelial cell adhesion molecule-1 (CD31), a scaffolding molecule for selected catenin family members whose binding is mediated by different tyrosine and serine/threonine phosphorylation. The Journal of biological chemistry. 2000;275:21435-43.

[66] Kawasaki Y, Imaizumi T, Matsuura H, Ohara S, Takano K, Suyama K, et al. Renal expression of alphasmooth muscle actin and c-Met in children with Henoch-Schönlein purpura nephritis. Pediatric Nephrology. 2008;23:913-9.

[67] Gerhardt H, Betsholtz C. Endothelial-pericyte interactions in angiogenesis. Cell and tissue research. 2003;314:15-23. 


\section{Figure Captions}

Figure 1. (A) Schematic diagram showing two independent crosslinking processes of the bioink, where alginate, GelMA, and 4-arm PEGTA are ionically and covalently crosslinked, respectively, upon exposure to $\mathrm{CaCl}_{2}$ solution and $\mathrm{UV}$ light. (B) Schematics showing the procedure of bioprinting perfusable hollow tubes with the cell-encapsulating blend bioink and subsequent vascular formation. (C) The designed multilayered coaxial nozzles and schematic diagram showing fabrication of perfusable hollow tubes with constant diameters and changeable sizes.

Figure 2. (A) Printability analysis of the blend bioinks. (B) Viscosity measurement and rheological behavior of 7\% GelMA, 3\% alginate, and 2\% PEGTA alone, as well as their mixtures at various concentrations at room temperature. The photographs on the right show the flow behavior of the respective bioinks at $10 \mathrm{~min}$ post transposing the containers. (C) Compressive moduli of bioinks composed of 7\% GelMA and 3\% alginate mixed with varied concentrations of 4-arm PEGTA ranging from $0-3 \%$ after exposure to $6.9 \mathrm{~mW} \mathrm{~cm}^{-2} \mathrm{UV}$ light for $30 \mathrm{~s}(\mathrm{n}=6 ; * p<0.05, * * p<0.001)$.

Figure 3. (A) Schematic diagram and representative fluorescence micrographs showing the bioprinted perfusable tubes displaying different outer diameters (I) and quantification data (II), as well as tubes with the same outer but different inner diameters (III). The insets in (I) and (II) are the cross-sectional views of the tube lumens. (B) Fluorescence photographs showing bioprinted perfusable tubes with various shapes, where green fluorescent beads were embedded into the walls during the bioprinting process; the insets are high-magnification images showing the perfusion of the lumens by red fluorescent beads. (C) Photographs showing single tubes bioprinted with a gradually increasing size (I) and periodically varying sizes (III); the high-magnification fluorescence micrographs in (II and IV) clearly show the varying diameters at selected locations indicated in (I and III), respectively.

Figure 4. (A) Schematics and corresponding fluorescence micrographs of the bioprinted 
tubular constructs with different aspect ratios of internal grids (I) and numbers of layers (II). (B) Confocal micrographs showing a uniform 3D structure composed of 10 layers of bioprinted tubes (containing green fluorescent beads), which were perfused with red fluorescent microbeads inside the lumens. (C) Fluorescence photographs before (inset) and after injection with red fluorescent microbeads into the lumen of the single, continuous bioprinted tube.

Figure 5. (A) Quantitative analysis of viable cells with different UV crosslinking times. (B) Fluorescence micrographs of Live/Dead staining after 1, 3, and 7 days of culture for the bioprinted tubes encapsulating vascular cells $\left(6.9 \mathrm{~mW} \mathrm{~cm} \mathrm{~cm}^{-2} \mathrm{UV}\right.$ light for $\left.30 \mathrm{~s}\right)$. (C) Measurement of cell metabolic activities of the bioprinted tubes under different UV crosslinking times. (D) Fluorescence micrographs showing f-actin/nuclei staining for the bioprinted constructs at $1,7,14$, and 21 days of culture $\left(6.9 \mathrm{~mW} \mathrm{~cm}^{-2} \mathrm{UV}\right.$ light for $\left.30 \mathrm{~s}\right)$. (E) Degradation profiles of the bioprinted constructs at different UV crosslinking times. $(n=6$; $* p<0.05)$

Figure 6. (A) Representative confocal micrographs of f-actin/nuclei staining after 21 days of culture post bioprinting $\left(6.9 \mathrm{~mW} \mathrm{~cm} \mathrm{~cm}^{-2} \mathrm{UV}\right.$ for $\left.30 \mathrm{~s}\right)$, showing different layers of the hollow fibers (I and II), longitudinal cross-section (III), and surface (IV) of the tubes, as well as 3D reconstruction indicating the perfusable vessel-like structure lined with vascular cells (V). (B) Confocal images of the vascular structure containing aSMA-expressing MSCs and CD31expressing HUVECs after 14 (I) and 21 (II) days of culture. 


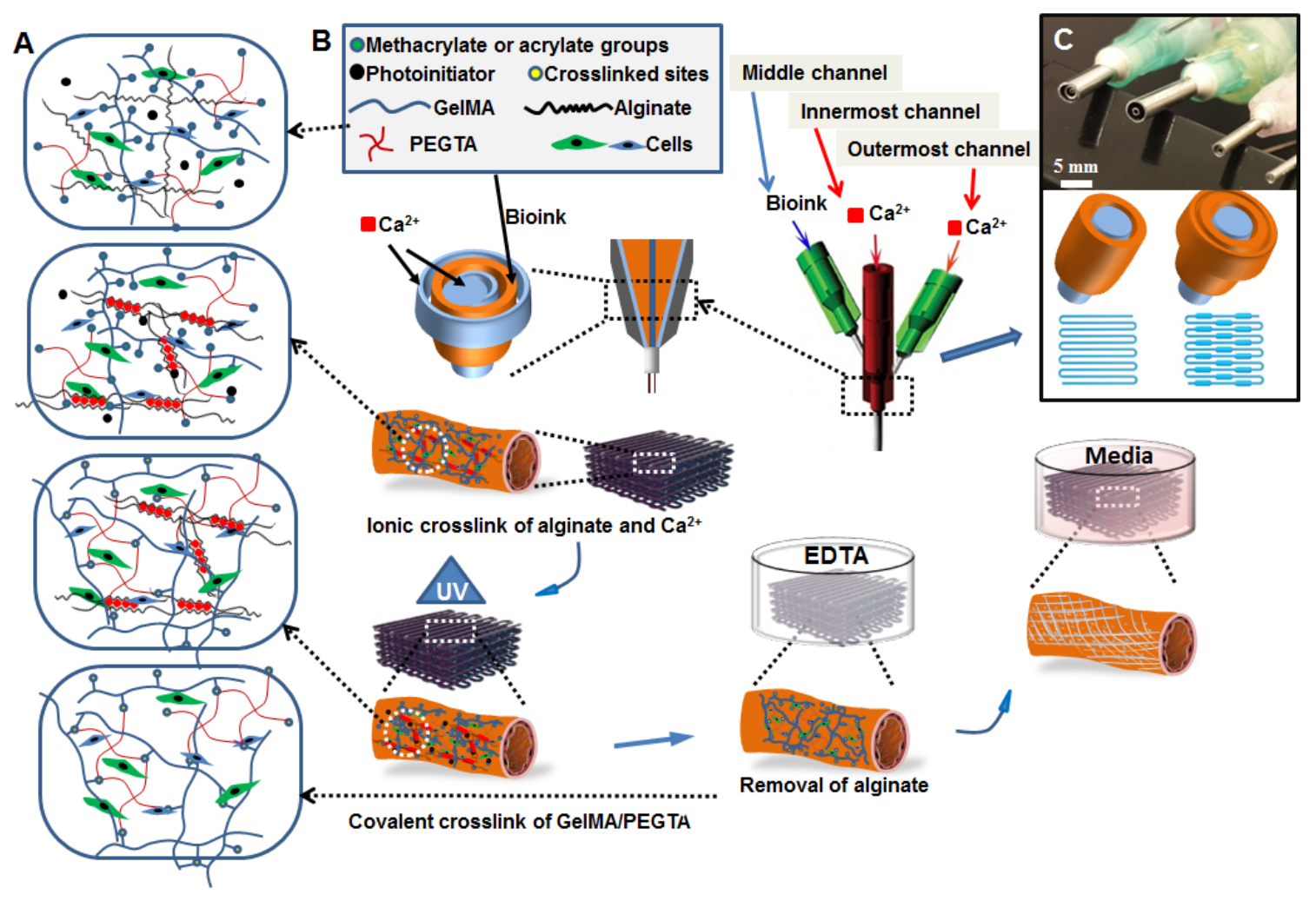

Figure 1. (A) Schematic diagram showing two independent crosslinking processes of the bioink, where alginate, GelMA, and 4-arm PEGTA are ionically and covalently crosslinked, respectively, upon exposure to $\mathrm{CaCl}_{2}$ solution and $\mathrm{UV}$ light. (B) Schematics showing the procedure of bioprinting perfusable hollow tubes with the cell-encapsulating blend bioink and subsequent vascular formation. (C) The designed multilayered coaxial nozzles and schematic diagram showing fabrication of perfusable hollow tubes with constant diameters and changeable sizes. 

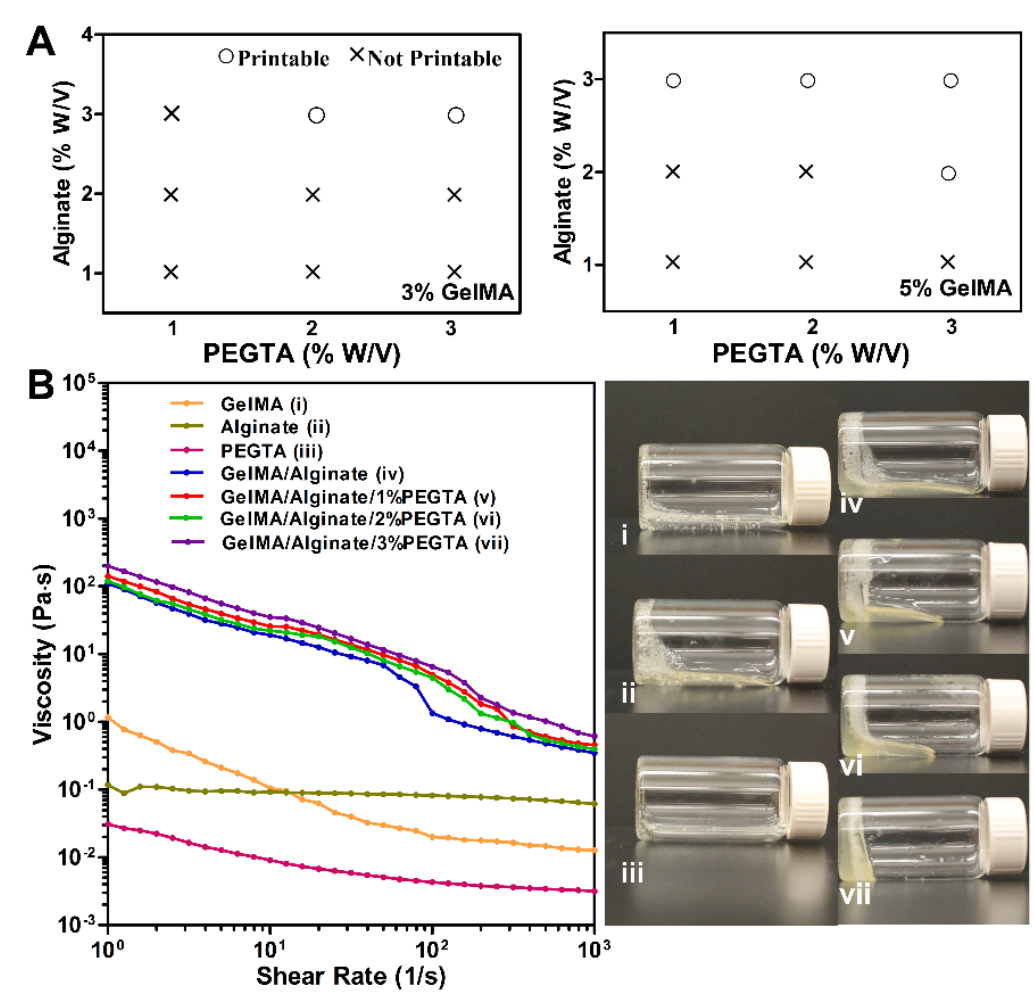
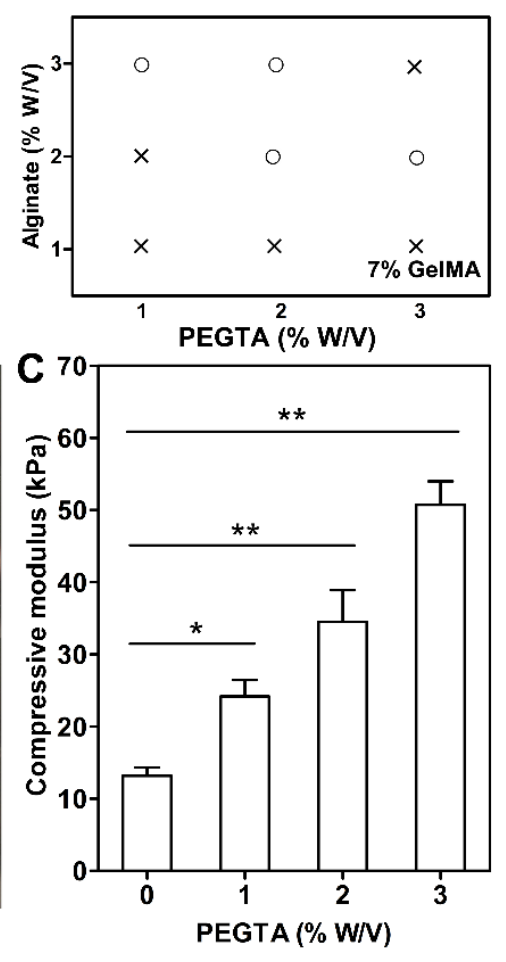

Figure 2. (A) Printability analysis of the blend bioinks. (B) Viscosity measurement and rheological behavior of 7\% GelMA, 3\% alginate, and 2\% PEGTA alone, as well as their mixtures at various concentrations at room temperature. The photographs on the right show the flow behavior of the respective bioinks at 10 min post transposing the containers. (C) Compressive moduli of bioinks composed of 7\% GelMA and 3\% alginate mixed with varied concentrations of 4-arm PEGTA ranging from $0-3 \%$ after exposure to $6.9 \mathrm{~mW} \mathrm{~cm}^{-2} \mathrm{UV}$ light for $30 \mathrm{~s}(\mathrm{n}=6 ; * p<0.05, * * p<0.001)$. 

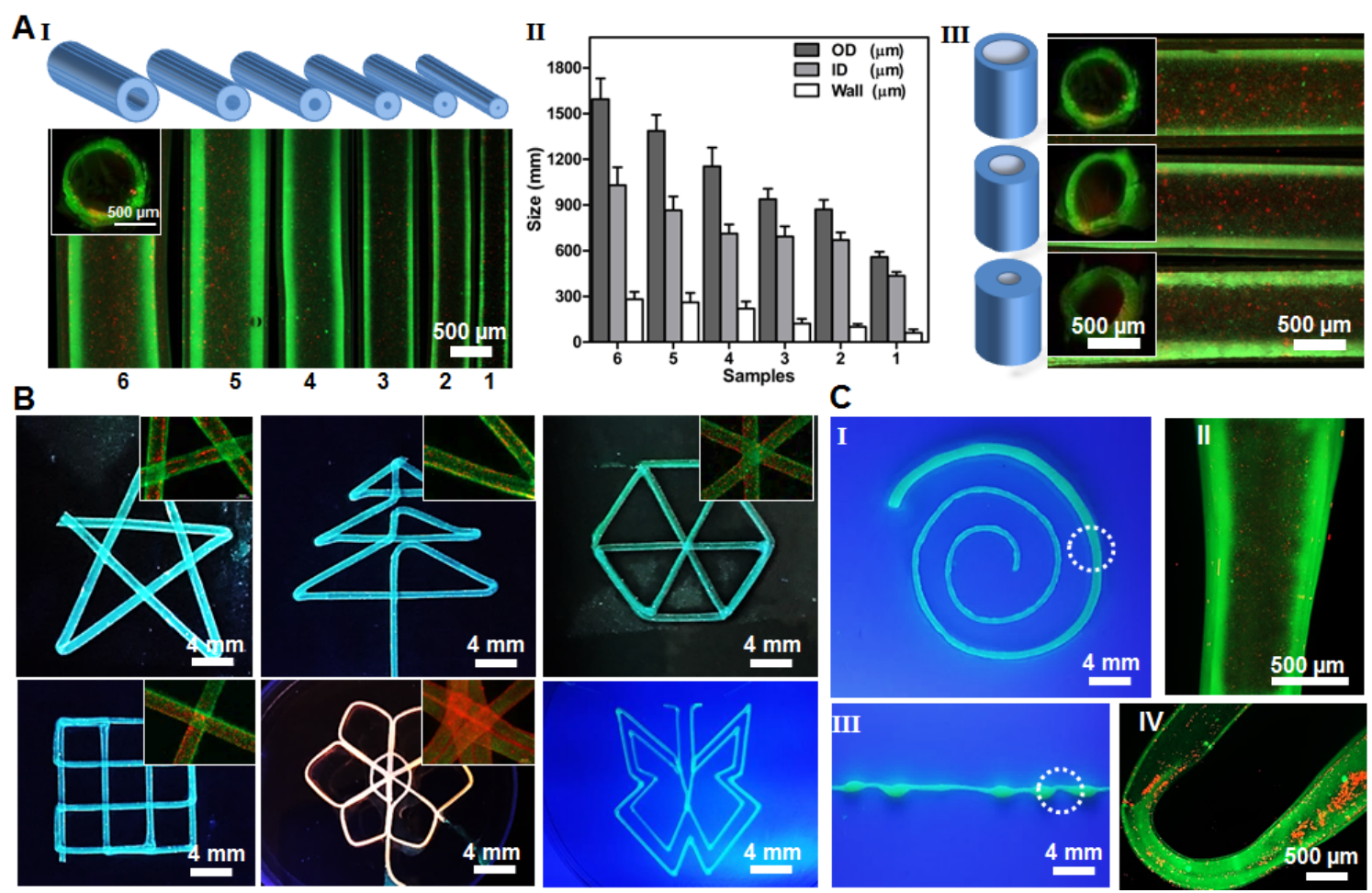

Figure 3. (A) Schematic diagram and representative fluorescence micrographs showing the bioprinted perfusable tubes displaying different outer diameters (I) and quantification data (II), as well as tubes with the same outer but different inner diameters (III). The insets in (I) and (II) are the cross-sectional views of the tube lumens. (B) Fluorescence photographs showing bioprinted perfusable tubes with various shapes, where green fluorescent beads were embedded into the walls during the bioprinting process; the insets are high-magnification images showing the perfusion of the lumens by red fluorescent beads. (C) Photographs showing single tubes bioprinted with a gradually increasing size (I) and periodically varying sizes (III); the high-magnification fluorescence micrographs in (II and IV) clearly show the varying diameters at selected locations indicated in (I and III), respectively. 

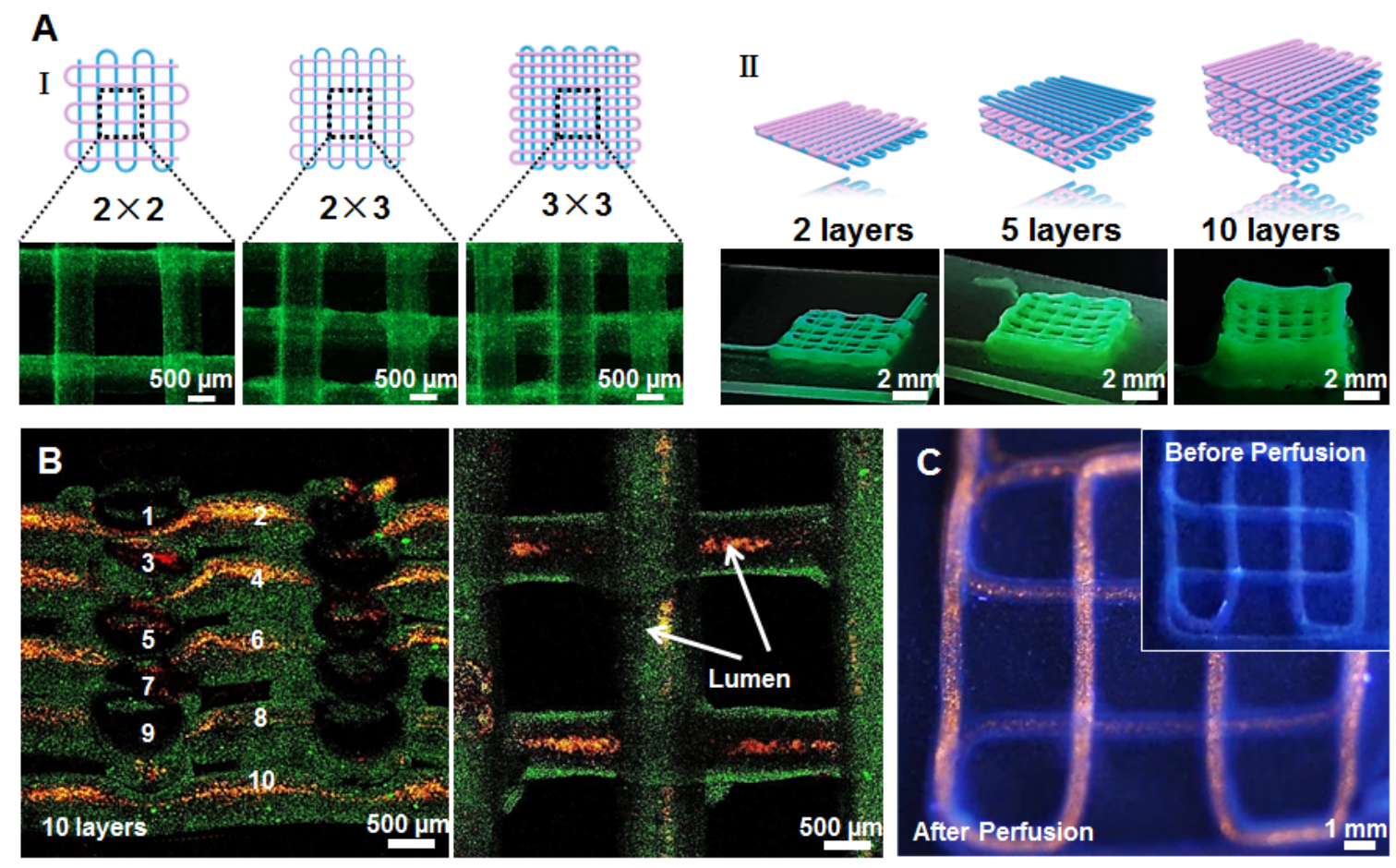

Figure 4. (A) Schematics and corresponding fluorescence micrographs of the bioprinted tubular constructs with different aspect ratios of internal grids (I) and numbers of layers (II). (B) Confocal micrographs showing a uniform 3D structure composed of 10 layers of bioprinted tubes (containing green fluorescent beads), which were perfused with red fluorescent microbeads inside the lumens. (C) Fluorescence photographs before (inset) and after injection with red fluorescent microbeads into the lumen of the single, continuous bioprinted tube. 

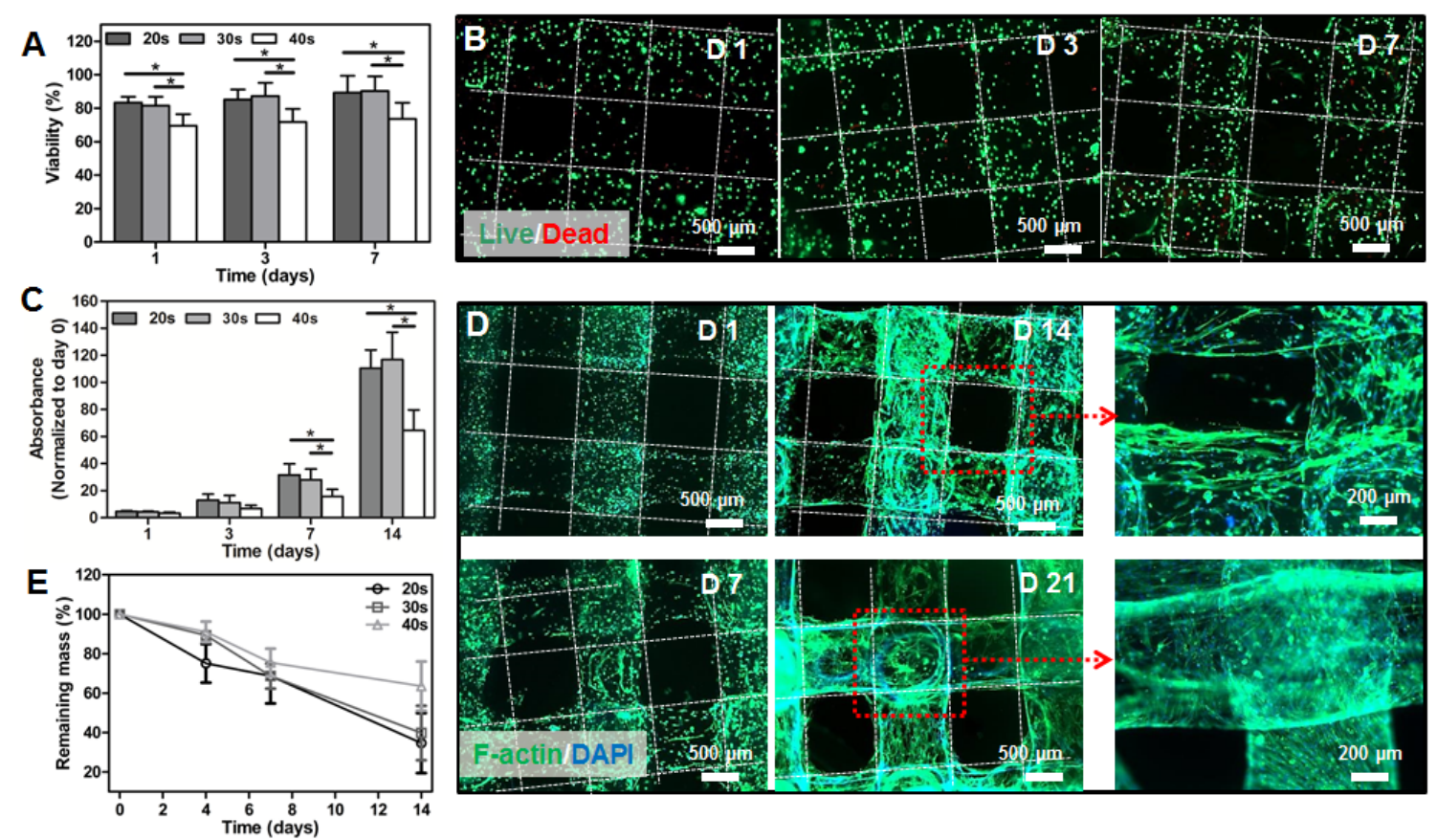

Figure 5. (A) Quantitative analysis of viable cells with different UV crosslinking times. (B) Fluorescence micrographs of Live/Dead staining after 1, 3, and 7 days of culture for the bioprinted tubes encapsulating vascular cells $\left(6.9 \mathrm{~mW} \mathrm{~cm}^{-2} \mathrm{UV}\right.$ light for $\left.30 \mathrm{~s}\right)$. (C) Measurement of cell metabolic activities of the bioprinted tubes under different UV crosslinking times. (D) Fluorescence micrographs showing f-actin/nuclei staining for the bioprinted constructs at $1,7,14$, and 21 days of culture $\left(6.9 \mathrm{~mW} \mathrm{~cm}^{-2} \mathrm{UV}\right.$ light for $\left.30 \mathrm{~s}\right)$. (E) Degradation profiles of the bioprinted constructs at different UV crosslinking times. $(n=6$; $* p<0.05)$ 

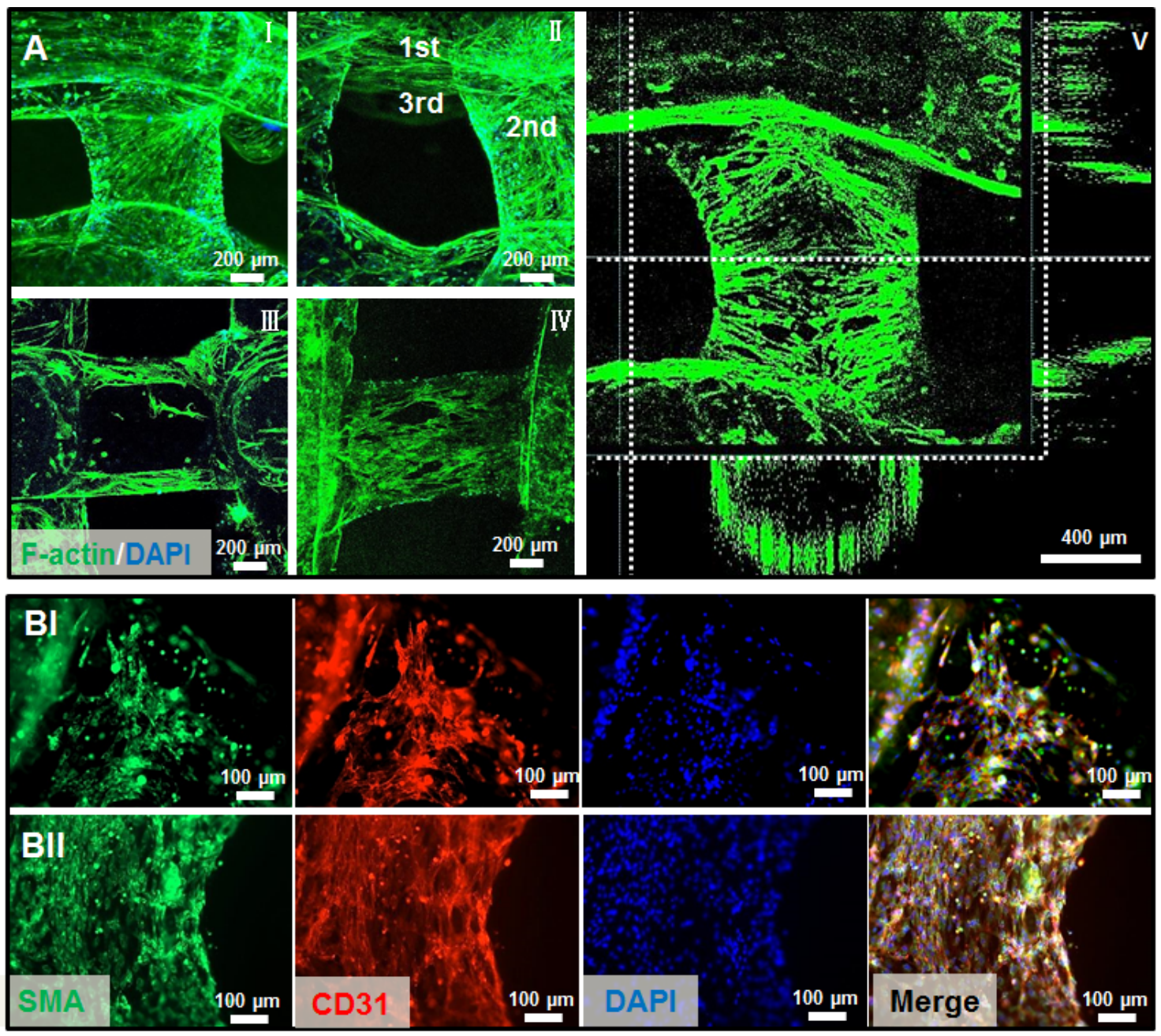

Figure 6. (A) Representative confocal micrographs of f-actin/nuclei staining after 21 days of culture post bioprinting $\left(6.9 \mathrm{~mW} \mathrm{~cm} \mathrm{mV}^{-2} \mathrm{UV}\right.$ for $\left.30 \mathrm{~s}\right)$, showing different layers of the hollow fibers (I and II), longitudinal cross-section (III), and surface (IV) of the tubes, as well as 3D reconstruction indicating the perfusable vessel-like structure lined with vascular cells (V). (B) Confocal images of the vascular structure containing aSMA-expressing MSCs and CD31expressing HUVECs after 14 (I) and 21 (II) days of culture. 